\title{
What Expands in an Expanding Universe?
}

\author{
JOSÉ A. DE FREITAS PACHECO \\ Observatoire de la Côte d'Azur - Laboratoire Lagrange, 06304 Nice Cedex, France \\ Manuscript received on December 15. 2014; accepted for publication on February 23, 2015
}

\begin{abstract}
In the present investigation, the possible effects of the expansion of the Universe on systems bonded either by gravitational or electromagnetic forces, are reconsidered. It will be shown that the acceleration (positive or negative) of the expanding background, is the determinant factor affecting planetary orbits and atomic sizes. In the presently accepted cosmology $(\Lambda \mathrm{CDM})$ all bonded systems are expanding at a decreasing rate that tends to be zero as the universe enters in a de Sitter phase. It is worth mentioning that the estimated expansion rates are rather small and they can be neglected for all practical purposes.
\end{abstract}

Key words: cosmic expansion, cosmology, cosmological models, gravitation.

\section{1-INTRODUCTION}

The nature of the cosmological expansion is a recurrent debate in cosmology. The most direct evidence for the expansion of the universe appeared with the first spectroscopic observations of extragalactic "nebulae" by Slipher (1915), which revealed that most of these objects have redshifted spectral features. Subsequent observations by Hubble (1929) put in evidence a relation between the distances of these "nebulae" and the observed redshift in their spectra. An interpretation of these observations was provided by Lemaitre (1931), who showed that an expanding relativistic universe model could account for the observed radial velocity of galaxies and, in particular, explaining the fact that the redshift increases with the distance. Nevertheless, since the discovery of the redshift phenomenon, alternative explanations to the cosmic expansion interpretation have been proposed. Zwicky (1929) hypothesized that photons lose energy by interacting with the intergalactic medium, the so-called "tired light" theory and its major difficulty is the mechanism behind the loss of energy. Arp (1988) studied different groups of galaxies and remarked on some of them having the presence of objects with discrepant radial velocities. These observations led him and Burbidge to argue that some redshifts might not be cosmological in origin (Burbidge 2001, Burbidge et al. 2006). With the discovery of type Ia supernovae (cosmological "candles") at moderate redshifts, it became clear that most of the alternative theories of the cosmic expansion are unable to explain the time stretching observed in the light curves of these events (Goldhaber et al. 2001).

E-mail: pacheco@oca.eu 
In the present investigation, the observed redshift of features present in the spectra of distant galaxies is considered as an evidence in favor of the cosmological expansion although, as we shall see later, it cannot be explained simply in terms of a Doppler effect.

A "classical" representation of the expansion of the Universe, frequently used in most scholar texts, was given by Misner et al. (1973) in their classical book "Gravitation". The universe is modeled by the surface of a rubber balloon covered with coins, which represent galaxies. As the balloon inflates, each coin may be considered to be the center of the expansion. The surface of the balloon represents the uniform stretching of space or the background. However, if space itself is stretching, does this means that everything in it is stretching? The aforementioned authors consider that "the coins themselves do not expand", meaning that the expansion would occur only at the scale of galaxies and clusters of galaxies but not at smaller scales. In other words, bodies hold together either by electromagnetic or gravitational forces, would not expand! However, if space is stretching how could these "bonded" systems not be affected? An early tentative of answering these questions was proposed by Dicke and Peebles (1964), who stated that if everything expands in the same proportion, then the expansion could not be detected since the "rules" also expand in the same proportion.

The concept of space in the general relativity theory (GRT) differs from that used in Newtonian physics or in the special theory of relativity. In the former, space is defined as a relation between particles while in the latter, space is not influenced by matter and exists independently of it. In Newtonian physics and special relativity, space has a mathematical character whereas in GRT it is more physical, since matter affects its geometry and dynamics, exemplified by the propagation of gravitational waves, the drag of a reference frame near a rotating body and the cosmic expansion itself. In GRT the geometry of the universe is described by the Friedmann-Robertson-Walker (FRW) metric which, in principle, does not specify any particular scale at which the expansion begins to occur.

Possible cosmic expansion effects on the solar system scale were studied more than 80 years ago by McVittie (1933), who did not reached any definite conclusion. He studied expansion effects on planetary orbits and, according to his calculations, the average orbital radius should remain constant. However, he used a particular coordinate system in which the physical interpretation of the results is not trivial. Jarnefelt $(1940,1942)$ reanalyzed this problem and confirmed that the planetary orbits do not participate in the expansion of the universe. A similar conclusion was also reached by Einstein and Straus (1945), who adopted a completely different approach. They showed that the Schwarzschild solution can be embedded in a dust-expanding universe and that test particles, representing planets, are unaffected by the expansion. This investigation has been criticized since the match between both metrics requires specific boundary conditions that are not fulfilled in the case of the solar system. After these negative results, Gautreau (1984) reinvestigated the problem of imbedding a Schwarzschild mass into a zero-curvature universe, and he concluded that orbits do expand. According to him, the cosmological fluid crosses a surface of constant $\mathbf{R}$, changing the amount of matter inside such a surface and affecting the planetary orbits, since test bodies experience a gravitational force that decreases with time. Anderson (1995) studied the motion of a pair of gravitating particles in an Einstein-de Sitter universe and he concluded that the expansion does affect the motion, excepting the case of pure circular orbits, which do not expand. This conclusion was confirmed by Cooperstock et al. (1998), who showed that the expansion affects all scales but, as one should expect, the magnitude of the effect is quite small. More recently, Sereno and Jetzer (2007) called the attention to a very important point: in reality planetary orbits are expanding but the effects on the orbital properties depend mainly on the acceleration (deceleration) of the expansion rather than on the expansion itself. 
In larger scales, i.e., in scales of clusters or superclusters of galaxies, expansion effects in these bonded structures were investigated by Noerdlinger and Petrosian (1971). They concluded that these structures expand at a rate that depends on the ratio of their average density to the background density.

In the atomic scale, the situation is also controversial. Bonnor (1996) obtained an exact solution of the Einstein-Maxwell equations for a particular case involving charged dust in equilibrium thanks to the balance between electrical and gravitational forces. According to Bonnor (1996) a body in this situation participates in the expansion. Later, Bonnor (1999) showed that in a de Sitter universe, the atomic radius remains strictly constant during the expansion, while in an Einstein-de Sitter universe the atom expands, but the amplitude of the effect is rather small. The effect of the expansion on atomic scales was reconsidered by Price (2005) who concluded that "either the atom expands or...completely ignores the expansion", a rather astonishing statement!

In the present paper, the question of what is expanding in a expanding universe is revisited and, in particular, we address the issue of how this expansion should be interpreted. The main conclusion is that all bodies suffer the consequences of the expansion of the universe but with different strengths. At atomic and solar system scales, effects are presently undetectable and, in practice, can be neglected. It is also shown that, in agreement with Sereno and Jetzer (2007), the acceleration (positive or negative) of the expansion is the dominant factor affecting the behavior of systems bonded gravitationally or electromagnetically. This paper is organized as follows: in Section 2 the interpretation of the receding motion of galaxies as a consequence of the expansion of space is revisited; in Section 3, expansion effects on planetary orbits are analyzed for different cosmological models; in Section 4, the consequences of the expansion for the size of atoms are discussed and, lastly, in Section 5 the main conclusions are given.

\section{2-THE RECEDING MOTION OF GALAXIES}

The redshift observed in the spectra of galaxies is commonly interpreted as a Doppler effect and as an evidence in favor of the cosmic expansion. The Hubble's law is a linear relation between the receding velocities and distances and, it is interesting to recall here the redshift-velocity relation for curved spaces (see, for instance, Harrison 1993) in order to emphasize the differences with the same relation derived for a Minkowski space. In the latter, the relativistic Doppler relation between the radial velocity and the redshift is well known and is given by:

$$
V(z)=\frac{c\left(2 z+z^{2}\right)}{\left(2+2 z+z^{2}\right)}
$$

This expression indicates that when $z \rightarrow \infty$, the radial velocity of the body tends to the light velocity and when $z<<1$, the velocity varies linearly with $z$, that is $V \approx c z$. Note that eq. 1 defines the physical velocity of the body that never becomes superluminal.

A flat, homogeneous and isotropic universe is described by the FRW metric, expressed in terms of the comoving coordinates $(r, \theta, \phi)$, i.e.,

$$
d s^{2}=-c^{2} d t^{2}+a^{2}(t)\left(d r^{2}+r^{2} d \theta^{2}+r^{2} \sin ^{2} \theta d \phi^{2}\right)
$$


where $a(t)$ is the scale factor. In this frame, receding galaxies are at rest if their peculiar velocities are zero. The proper distance $R$ to an object, measured at the instant $t$ is $R(t)=a(t) r$, where the comoving radial coordinate satisfies

$$
r=c \int_{t_{e}}^{t} \frac{d t^{\prime}}{a\left(t^{\prime}\right)}
$$

In the integral above, $t_{e}$ is the proper time at the instant of the emission of a photon that is received by the observer at the instant $t$. This integral, using the definition of the Hubble parameter that is, $H=\dot{a} / a$, and of the cosmic redshift, $1 / a\left(t_{e}\right)=(1+z)$, can be recast as (note that, in general, the present value of the scale parameter is taken to be one, i.e., $\left.a\left(t_{0}\right)=1\right)$

$$
r=c \int_{0}^{z} \frac{d z^{\prime}}{H\left(z^{\prime}\right)}
$$

The time derivative of the proper distance $R$ gives

$$
\frac{d R}{d t}=r \frac{d a}{d t}+a \frac{d r}{d t}=H R+a \frac{d r}{d t}=V_{H}+V_{p}
$$

where $V_{H}=H R$ is the receding velocity at the proper distance $R$ (Hubble's law) and $V_{p}=a(t)(d r / d t)$ is the peculiar velocity of the test galaxy. Using eq. 4 one obtains for the receding velocity as a function of the redshift

$$
V_{H}=H_{0} c \int_{0}^{z} \frac{d z^{\prime}}{H\left(z^{\prime}\right)}
$$

In this equation, the receding velocity is that seen by an observer at the present time $t_{0}$, when $a\left(t_{0}\right)=1$. As an example, let us calculate the redshift-velocity relation for a "dusty" Einstein-de Sitter model. In this case, from Einstein's equations, one obtains for the Hubble parameter $H(z)=H_{0}(1+z)^{3 / 2}$. Replacing into eq. 6 one obtains

$$
V_{H}=2 c\left[1-(1+z)^{-1 / 2}\right]
$$

For $z<<1$ this equation gives $V_{H} \approx c z$ but at high redshifts the receding velocity differs considerably from eq. 1. Objects located near the particle horizon $r_{p}=2 c / H_{0}$ are receding with velocities higher than $c$. This result does not mean that a body is moving with a superluminal velocity but it simply indicates the rate at which the space between the observer and the considered galaxy is expanding (see Page's 1993 criticism to this interpretation).

A comparison of eq. 7 with eq. 1 shows differences that appear clearly when both equations are compared at small redshifts. At a first order in $z$ they coincide but when the expansion is performed up to the second order, Gr $\phi$ n and Elgar $\phi y$ (2007) showed that the cosmological redshift is given by the sum 
of two contributions: the first is a pure Doppler effect in the Minkowiski space resulting from the series expansion of eq. 1 and the second is a pure gravitational effect, produced by the mass of the accelerated (or decelerated) cosmic fluid embedded by a spherical surface with a comoving radius $r(z)$.

\section{1-REVISITING THE TETHERED GALAXY PROBLEM}

The fact that galaxies are receding is often interpreted as "an expansion of space itself among galaxies". However, this view has been challenged by different authors, who argued that the expansion of space is a "peculiarity" of the adopted coordinate system, since the expansion could equally well be described as an "expansion through space" .

The expansion of galaxies is known as the "Hubble flow" and the general belief is that if a galaxy is set up at rest with respect to the observer and then released, it will start to recede as it merges into the Hubble flow. In fact, Peacock $(1999,2002)$ and Whiting (2004) had imagined a "thought experiment" in which the test galaxy has initially a non-zero peculiar velocity and in which the solution of the equations of motion indicates a trajectory rather different from that one would expect based on the "common sense".

In their experiment, a galaxy (our galaxy, for instance, where the observer is situated) located at the origin of the coordinate system is rigidly attached to another test galaxy by a tether. Under this condition, its proper (or physical) distance $R$ remains constant and its proper velocity $d R / d t$ with respect to the observer, is zero. Note that the initial condition for the tethered galaxy implies that its peculiar velocity is different from zero and must be equal and opposite to the Hubble flow velocity (see eq. 5). Since the proper distance is constant while the tether is present, the comoving distance of the test galaxy decreases. If the tether is then suddenly suppressed, the test galaxy has the following trajectory in comoving coordinates: first it approaches the observer (negative peculiar velocity), then crosses the origin (observer position) and joins asymptotically the Hubble flow on the opposite side of the sky.

Peacock $(1999,2002)$ and Whiting $(2004)$ argued that the fact that the test galaxy joins the Hubble flow only asymptotically and on the opposite side of the observer, is contrary to what one would expect for a test body dropped into the expanding background. This unexpected result led them to question the idea of an "expanding background". However, Davis et al. (2003) consider that this behavior is consistent with the idea that space is expanding, being a consequence of the acceleration of the expansion and not the consequence of any kind of "drag-force" associated with the expansion itself. In other words, this occurs because the proper acceleration of the test galaxy, once free from the tether, depends only on the acceleration of the background. In fact, this interpretation was contested by Gr $\phi$ n and Elgar $\phi y$ (2007), who emphasized that Davis et al. (2003), in order to reach such a conclusion, assumed that the peculiar velocity of the test galaxy decays as the inverse of the scale factor without solving the geodesic equation. In reality, as we shall see below, the peculiar velocity decays as $1 / a(t)$ but only at late phases of the trajectory, when the test galaxy approaches asymptotically the Hubble flow.

The evolution of the radial comoving coordinate is governed by the geodesic equation

$$
\frac{d^{2} r}{d t^{2}}+\frac{2}{a} \frac{d a}{d t} \frac{d r}{d t}-\frac{a}{c^{2}} \frac{d a}{d t}\left(\frac{d r}{d t}\right)^{3}=0
$$

A first integration of this equation can be done by defining the new variable $y=d r / d t$ and using the scale factor as the independent variable such as $d / d t=\dot{a}(d / d a)$. Under these conditions eq. 8 can be recast as 


$$
\frac{d y}{d a}+\frac{2}{a} y-\frac{a}{c^{2}} y^{3}=0
$$

This equation can be easily integrated and, recalling the definition of the peculiar velocity that is; $V_{p}=$ ay, one obtains

$$
V_{p}(a)= \pm \frac{c}{\sqrt{1+k c^{2} a^{2}}}
$$

where $k$ is an integration constant. Note that the solution can be either positive or negative. The signal and the integration constant, are fixed by the initial conditions. If the test galaxy is released at rest $\left(V_{p}=0\right)$, it immediately joins the Hubble flow, since its comoving radial coordinate remains constant. In the particular case of the tethered galaxy, one imposes that when $a=a_{*}$ (instant at which the tether is suppressed) the peculiar velocity is $V_{p}\left(a_{*}\right)=-V_{H}\left(a_{*}\right)=-V_{H_{*}}$. Under this condition, one obtains

$$
k c^{2}=\frac{1}{a_{*}^{2}}\left(\frac{c^{2}}{V_{H_{*}}^{2}}-1\right)
$$

Thus, the peculiar velocity evolves as

$$
V_{p}(a)=-\frac{c}{\sqrt{1+\frac{a^{2}}{a_{*}^{2}}\left(\frac{c^{2}}{V_{H_{*}}^{2}}-1\right)}}
$$

a relation valid for $a \geq a_{*}$. This equation says that whatever is the acceleration of the cosmic expansion, the initial direction of the peculiar velocity remains unchanged but the effects of the expansion of space itself can be seen at late times, when $a(t) \gg a_{*}$ and when the peculiar velocity decays as the inverse of the scale factor, joining asymptotically the Hubble flow, as one should expect. Thus, no inconsistency with the space expansion interpretation exists. Note also that eq. 11 requires that the test galaxy be located necessarily inside the Hubble radius or that the receding velocity at the initial position be less than $c$. It should be emphasized that galaxies in the Hubble flow do not feel any force. Hence, the image of the expansion acting like a "drag-force" is not adequate.

Moreover, we must be very careful when analyzing such a "thought experiment". The FRW metric describes a homogeneous universe but the introduction of an observer breaks such a homogeneity. Let us explain in more details the flaw in the interpretation of the aforementioned experiment. Imagine that another observer, in a different galaxy, decides to perform the same experiment. If this second observer attaches a tether to the same test galaxy, it is easy to verify the impossibility to satisfy the same initial conditions for both observers. This means that the condition of isotropy and homogeneity are locally broken and the experiment is valid only for a given observer. 


\section{3-PLANETARY ORBITS: EXPANSION OR CONTRACTION?}

As we have previously mentioned, the question concerning whether the cosmic expansion affects (or not) the planetary orbits still has controversial answers. One of the difficulties is that the standard cosmological picture does not describe the gravitational field near a star like the Sun. In the vicinity of the Sun, the space geometry is described by the Schwarzschild metric and the cosmological field probably only exerts a perturbative effect. The match between both metrics (Schwarzschild and cosmological) was investigated in the past by McVittie (1933), by using an approximate approach, and by Einstein and Straus (1945), Schucking (1954), Bonnor (2000) among others.

The problem of imbedding a point mass into cosmology was also considered by Gautreau (1984) from a different point of view. According to him, the mass contributing to the gravitational forces is the MisnerSharp mass inside the sphere whose radius is the orbital distance of the test particle (planet), that is

$$
M=M_{\odot}+\frac{4 \pi}{3} \rho(t) R^{3}
$$

As the universe expands, the flow across the sphere $R=$ constant, decreases the Misner-Sharp mass, weakening the gravitational force on the planet and causing modifications in its orbit.

We adopt here a semi-Newtonian approach to compute the effects of the cosmic expansion on planetary orbits, guided by the basic ideas of Gautreau (1984) but instead of using the Misner-Sharp mass, we used a modified Poisson equation (Peebles 1980) that includes the contribution of pressure to the sources of gravitation, that is

$$
\nabla^{2} \Phi=4 \pi G M_{\odot} \delta(r)+\frac{4 \pi G}{c^{2}}(\varepsilon+3 P)
$$

where, on the right side, the first term is the contribution from a point-source (the Sun) and the second term is the contribution due to the cosmological fluid inside the orbit. Integration of eq.14 gives for the gradient of the potential

$$
\frac{d \Phi}{d R}=\frac{G M_{\odot}}{R^{2}}+\frac{4 \pi G}{3 c^{2}}(\varepsilon+3 P) R
$$

Note that the second term on the right side of the above equation can be replaced by the Friedmann equation involving the acceleration of the scale factor, i.e.,

$$
\frac{d \Phi}{d R}=\frac{G M_{\odot}}{R^{2}}-\frac{\ddot{a}}{a} R
$$

The radial (physical) coordinate $R$ in the orbital plane obeys the equation

$$
\frac{d^{2} R}{d t^{2}}=-\frac{d \Phi}{d R}+\frac{V_{t}^{2}}{R}
$$


where $V_{t}$ is the tangential velocity such as, $J=R V_{t}$ is the specific angular momentum of the test particle, a conserved quantity (note that the presence of the cosmological term in eq. 16, which depends on time, implies that the energy is not strictly conserved on scales of the Hubble time). Considering the second term on the right side of eq. 16 as a small perturbation, the polar equation of the unperturbed orbit is

$$
R=\frac{A\left(1-e^{2}\right)}{(1+e \cos \theta)}
$$

where $A$ is the semi-major axis and $e$ is the eccentricity. Deriving twice with respect to time this equation and taking into account the angular momentum conservation permit to express the radial acceleration as

$$
\frac{d^{2} R}{d t^{2}}=-\frac{e \cos \theta}{A\left(1-e^{2}\right)} V_{t}^{2}
$$

Combining this result with eqs.17 and 16, after some algebra, one obtains

$$
V_{t}^{2}=\frac{G M_{\odot}}{A\left(1-e^{2}\right)}\left[\frac{(1-e \cos \theta)^{2}}{(1+2 e \cos \theta)}\right]-\frac{\ddot{a}}{a} \frac{A^{2}\left(1-e^{2}\right)^{2}}{(1+e \cos \theta)(1+2 e \cos \theta)}
$$

In the equation above, dynamical quantities vary with two different timescales: a short and periodic one, due to the orbital motion, and a long one due to the cosmic expansion. Since we are interested in the long term variation of the semi-major axis due to cosmological effects, let us perform an average of eq. 20 over one orbital period. For a Keplerian motion, the average of any dynamical function $F(\theta)$ is given by

$$
\langle F\rangle=\frac{\left(1-e^{2}\right)^{3 / 2}}{2 \pi} \int_{0}^{2 \pi} \frac{F(\theta) d \theta}{(1+e \cos \theta)^{2}}
$$

Performing a term by term average of eq. 20 and at the end, retaining only up to second order terms in the eccentricity, one obtains

$$
\left\langle V_{t}^{2}\right\rangle=\frac{G M_{\odot}}{A}\left(1+\frac{3}{2} e^{2}\right)-\frac{\ddot{a}}{a} A^{2}\left(1+\frac{9}{2} e^{2}\right)
$$

Deriving the equation above with respect to time and, for the moment, neglecting the small long term variation of the eccentricity one gets

$$
\frac{d\left\langle V_{t}^{2}\right\rangle}{d t}=-\frac{G M_{\odot}}{A^{2}} \frac{d A}{d t}\left(1+\frac{3}{2} e^{2}\right)-\frac{d}{d t}\left(\frac{\ddot{a}}{a} A^{2}\right)\left(1+\frac{9}{2} e^{2}\right)
$$

Use the angular momentum conservation to obtain

$$
\left\langle V_{t}^{2}\right\rangle=\frac{J^{2}}{A^{2}}\left(1-e^{2}\right)^{-1 / 2}
$$


and, by derivation

$$
\frac{d\left\langle V_{t}^{2}\right\rangle}{d t}=-\frac{2\left\langle V_{t}^{2}\right\rangle}{A} \frac{d A}{d t}
$$

Combining eqs. 22, 23 and 25, after some algebra and using Kepler's third law, one obtains

$$
\frac{1}{A} \frac{d A}{d t}=\frac{P^{2}}{4 \pi^{2}}\left(1+3 e^{2}\right) \frac{d}{d t}\left(\frac{\ddot{a}}{a}\right)
$$

where here $P$ is the orbital period of the considered planet.

Equation 26 indicates that the acceleration of the expansion is the main factor affecting the planetary orbits and not the expansion itself, in agreement with the past conclusion by Sereno and Jetzer (2007), although the factor depending on the eccentricity is not exactly the same as that derived by those authors. Note also that eq. 26 differs from the result obtained by Gautreau (1984), who concluded that $d \lg A / d t \propto P^{2} H^{3}$. Gautreau's relation implies that the orbit is affected by the expansion rate, measured by the Hubble parameter, and not by the acceleration of the expansion. Consequently, in his approach the effects are the same, either the expansion is accelerated or decelerated.

In the case of a de Sitter cosmology, $\ddot{a} / a=H_{0}^{2}$ and, consequently, $d \lg A / d t=0$. In other words, despite the cosmic expansion being accelerated, the orbit is not affected. This can be understood by the fact that the energy density due to vacuum inside the orbit of the planet remains constant during the expansion and hence, it does not produce any long term variation on the gravitational forces acting on the test body.

For a cosmic fluid with an equation of state $P=w \varepsilon$, the relative variation of the semi-major axis is given by

$$
\frac{d \lg A}{d t}=\frac{P^{2}}{9 \pi^{2}}\left(1+3 e^{2}\right) \frac{(1+3 w)}{(1+w)^{2} t^{3}}
$$

The above equation is valid if $w>-1$. For the cases $w=0$ ("dust" equation of state) or $w=1 / 3$ (relativistic fluid), we have $d \lg A / d t>0$. Thus, for these models in which the expansion is decelerated, the orbit expands because the energy density and the pressure of the cosmic fluid decreases inside the orbit. Note also that the expansion rate decreases with time and, for instance, in the case of the Einstein-de Sitter model $(w=0)$, the orbit of the Earth would be presently increasing at a rate of about $6.4 \times 10^{-20} \mathrm{~cm} / \mathrm{yr}$. It is worth mentioning that Dirac (1979) modified the Schwarzschild metric in order to fit with the Einstein-de Sitter model, concluding that planetary orbits spiral inwards, in opposition to the present result.

If $w>-1 / 3$, planetary orbits will always be expanding since $\varepsilon+3 P>0$. However, if the equation of state parameter is in the range $-1<w<-1 / 3$, the case of quintessence models having an accelerated expansion, the situation is different since $d \lg A / d t<0$ or, in other words, the orbits are shrinking. The reason for this opposite behavior is the following: despite the energy density being positive, in the quintessence domain the negative pressure term prevails as a gravitational source in the second term on the right side of eq. 14 . This negative pressure acts like a "repulsive" gravity and since it decreases with time, the orbit contracts. If $w<-1$ we have a "phantom" fluid because the energy density increases as the universe expands. Phantom 
models have a future singularity at $t=t_{s}$ (the so-called "Big Rip") and a shrinking event horizon, that is, $R_{H} \propto c\left(t_{s}-t\right)$. The scale factor increases as $a \propto 1 /\left(t_{s}-t\right)^{n}$, where $n=-2 /[3(1+w)]$, leading to a relative variation rate of the semi-major axis given by

$$
\frac{d \lg A}{d t}=-\frac{P^{2}}{9 \pi^{2}}\left(1+3 e^{2}\right) \frac{(1+3 w)}{(1+w)^{2}\left(t_{s}-t\right)^{3}}
$$

and hence $d \lg A / d t>0$ for $w<-1$. Contrary to the quintessence case, in phantom models the energy density and the negative pressure increase as the Universe evolves, causing the expansion of the planetary orbits. Notice that as the "Big Rip" singularity is approached, the expansion rate of the orbit increases considerably leading to a disruption of all planetary systems in the universe.

In the $\Lambda$ CDM model, the scale factor varies as

$$
a(t)=\left(\frac{\Omega_{m}}{1-\Omega_{m}}\right)^{1 / 3} \sinh ^{2 / 3}\left(\frac{3 \sqrt{1-\Omega_{m}}}{2} H_{0} t\right)
$$

where $\Omega_{m}$ is the matter density parameter. In this case, it is trivial to show that $d \lg A / d t>0$ and therefore the orbits expand with a decreasing rate, which tends to zero as the model approaches the de Sitter cosmology. This is because the gravitational source term, $(\rho+3 P)$, decreases inside the orbit as the universe expands, producing a behavior similar to Einstein-de Sitter. As the energy related to the cosmological constant becomes dominant, the expansion rate tends to zero since $\ddot{a} / a \rightarrow 0$.

\section{4-EXPANSION EFFECTS ON ATOMS}

Past investigations on possible effects of the cosmic expansion at atomic scales were based on the solution of the Maxwell equations in a FRW background. The atom was modeled as a "mini-solar system", that is an electron orbiting, in the equatorial plane and a massive nucleus at rest positioned at the origin (Bonnor 1999, Price 2005). In these studies deviations from circular or closed orbits were considered in different cosmologies, in particular the de Sitter and the Einstein-de Sitter models.

However, atomic systems are quantized or in other words, the energy and the angular momentum have a discrete spectrum of values. In our approach we assume that the effects of the cosmic expansion can be considered as a small perturbation on the energy levels of the atom and that the perturbing potential is (see eq. 16)

$$
\delta \Phi_{p}=-\frac{1}{2}\left(\frac{\ddot{a}}{a}\right) R^{2}
$$

Using the quantum perturbation theory, the change in the energy eigenvalue of the fundamental state is (Merzbacher 1961)

$$
\Delta E_{0}=\int \Psi_{0}\left(\delta \Phi_{p}\right) \Psi_{0} d V
$$


The equation above says that the change in the energy of the ground level is approximately given by the expectation value of the perturbation in the unperturbed eigenstate. Using an approximate wave function that reproduces correctly, through a variational approach, the ground level energy of hydrogen, one obtains from eqs. 30 and 31

$$
\Delta E_{0}=\frac{3}{4} \frac{\hbar^{2}}{I_{H}^{2}} \frac{d}{d t}\left(\frac{\ddot{a}}{a}\right)
$$

where $I_{H}$ is the energy of the fundamental level. Since the energy of the ground state varies as $I_{H} \propto-1 / A_{B}$, where $A_{B}$ is the Bohr radius, the variation rate of the size of the hydrogen atom is

$$
\frac{d \lg A_{B}}{d t}=\frac{3}{4} \frac{\hbar^{2}}{I_{H}^{2}} \frac{d}{d t}\left(\frac{\ddot{a}}{a}\right)
$$

Equation 33 has the same structure as eq. 26, that is, it depends on the variation rate of the acceleration of the cosmic expansion. Thus, the size of the atom has the same behavior as that of planetary orbits. No effect is expected for the de Sitter cosmology, in agreement with the conclusions by Bonnor (1999). Atoms expand in the case of the Einstein-de Sitter model and for cosmologies in which the equation of state parameter $w$ is higher than $-1 / 3$. For quintessence models satisfying $-1<w<-1 / 3$, the atom suffers a contraction and expands if $w<-1$, case of phantom cosmologies. As a numerical example, the present relative variation rate of the Bohr radius for the Einstein-de Sitter model is $d \lg A_{B} / d t=3 \times 10^{-64} y r^{-1}$, a negligible amount that led Bonnor (1999) to conclude that, for practical purposes, atoms do not expand.

\section{5-CONCLUSIONS}

In the "thought experiment" of a tethered galaxy, the trajectory followed by the test galaxy is considered by some authors as an argument against the interpretation of the Hubble flow as an expansion of space. We have shown that the solution of the radial geodesic equation depends on the initial conditions imposed to the peculiar velocity of the test galaxy. If the test body is dropped into the background with a zero peculiar velocity, then it immediately joins the Hubble flow. On the one hand, if its peculiar velocity is non-zero, the trajectory depends on the initial direction of the motion. In the case of the tethered galaxy, the initial peculiar velocity is negative (opposite to the Hubble flow) and the galaxy, once free from the tether, moves in the direction of the observer, crosses the origin and joins asymptotically the Hubble flow in the opposite side of the sky. On the other hand, if the initial direction is positive, the test galaxy moves away and joins asymptotically the Hubble flow far from its initial position. The expansion of the background produces the well-known decay of the peculiar velocity that is proportional to the inverse of the scale factor. Thus, there is no contradiction with the interpretation of the Hubble flow as an expansion of space.

Planetary orbits and size of atoms are affected by the expansion in the same way, since expansion or contraction rates depend on the acceleration rate of the expansion itself. In our approach, expansion effects on planetary orbits were computed by using a semi-Newtonian formalism, leading to a modification of the Poisson equation, which includes the contribution of the cosmic fluid pressure in the source term. Atoms 
are quantum systems and the cosmic expansion acts like a perturbation that modifies the energy levels, in particular the ground state and the mean distance of electrons with respect to the atomic nucleus.

In agreement with previous investigations, in a de Sitter cosmology planetary orbits and the size of atoms remain unchanged. However, for the Einstein-de Sitter cosmology, either with a "dust" or a "radiation" equation of state, planetary orbits and atomic sizes increase with time but a decreasing rate. On the contrary, in quintessence models in which the conditions $\varepsilon+3 P<0$ and $\varepsilon+P>0$ are satisfied, planetary orbits and atomic sizes shrink. This is not the case if the condition $\varepsilon+P>0$ is violated (phantom cosmology) since an opposite behavior is expected. In phantom models, planetary orbits and atomic sizes increase and all bonded systems are disrupted near the future singularity present in these cosmologies. For the present accepted model, the $\Lambda \mathrm{CDM}$ cosmology, orbits and atomic sizes are expanding at a decreasing rate, which tends to zero as the expansion enters in a de Sitter phase.

In conclusion, in an expanding universe everything expands (or contracts) but at different rates, according to the interactions that hold the different bodies together. In general the expansion (contraction) rates are quite small and can be neglected for all practical purposes.

\section{RESUMO}

Neste artigo, os efeitos possíveis da expansão do Universo em sistemas ligados por forças gravitacionais ou eletromagnéticas são reconsiderados- Será demonstrado que a aceleração (positiva ou negativa) de expansão é o fator determinante que afeta as órbitas planetárias e as dimensões atômicas. Na cosmologia atualmente aceita (ACDM) todos os sistemas ligados estão em expansão com uma taxa decrescente que tende a zero à medida que o Universo entra na fase de Sitter. Vale a pena mencionar que as taxas de expansão estimadas são muito pequenas e podem ser desprezadas para todos os fins práticos.

Palavras-chave: expansão cósmica, cosmologia, modelos cosmológicos, gravitação.

\section{REFERENCES}

ANDERSON JL. 1995. Multiparticle dynamics in an expanding universe. Phys Rev Lett 75: 3602-3604.

ARP HC. 1988. Quasars, Redshifts and Controversies, Cambridge University Press.

BONNOR WB. 1996. The cosmic expansion and local dynamics. Mon Not R Astron Soc 282: 1467-1469.

BONNOR WB. 1999. Size of a hydrogen atom in the expanding universe. Classical Quant Grav 16: 1313-1321.

BONNOR WB. 2000. A generalization of the Einstein-Straus vacuole. Classical Quant Grav 17: 2739-2748.

Burbidge G. 2001. Noncosmological Redshifts. Pub Astron Soc Pac 113: 899-902.

BURBIDGE G, BURBIDGE EM, ARP HC AND NAPIER WM. 2006, arXiv:0605140.

CAldwell RR, Kamionkowski M And Weinberg NN. 2003. Phantom Energy: Dark Energy with $w<-1$ Causes a Cosmic Doomsday. Phys Rev Lett 91, \# 071301.

COOPERSTOCK FI, FARAONI V AND VolLicK DN. 1998. The Influence of the Cosmological Expansion on Local Systems. Astrophys J 503: 61-66.

DAVIS TM, LINEWEAVER CH AND WEBB JK. 2003. Solutions to the tethered galaxy problem in an expanding universe and the observation of receding blueshift objects. Am J Phys 71: 358-364.

DICKE RH AND PEEBLES PJE. 1964. Evolution of the Solar System and the Expansion of the Universe. Phys Rev Lett 12: $435-437$. DIRAC PAM. 1979. The Large Numbers hypothesis and the Einstein theory of gravitation. Proc Roy Soc Lond A365: 19-30.

EINSTEIN A AND STRAUS EG. 1945. The Influence of the Expansion of Space on Gravitational Fields Surrounding the Individual Stars. Rev Mod Physics 17: 120-124.

GAUTREAU R. 1984. Imbedding a Schwarzschild mass into cosmology. Phys Rev D29: 198-206.

GoldhaBeR ET AL. 2001. Timescale Stretch Parametrization of Type Ia Supernova B-Band Lightcurves. Astrophys J 558: 359386.

GR $\Phi$ N $\Phi$ AND ELGAR $\Phi$ Y $\Phi .2007$. Is space expanding in the Friedmann universe models? Am J Phys 75: 151-157, arXiv:0603162. 
HARRISON E. 1993. The Redshift-Distance and Velocity-Distance Laws. Astrophys J 403: 28-31.

HUBBLE E. 1929. A Relation between Distance and Radial Velocity among Extra-Galactic Nebulae. P Natl Acad Sci 15: $168-173$.

JANERFELT G. 1940. Zum Einkorperproblem in dem sich ausdehnenden Raume. Ann Acad Sci Fenn A55(3): 21.

JANERFELT G. 1942. Das Einkorperproblem in dem ausdehnendem Raume der Einstein-de Sitterschen Welt. Ann Acad Sci Fenn A1(12): 38.

LEMAÎTRE G. 1931. Expansion of the Universe: A homogeneous universe of constant mass and increasing radius accounting for the radial velocity of extra-galactic nebulae. Mon Not R Astron Soc 91: 483-490.

MCViTtie GC. 1933. The mass-particle in an expanding universe. Mon Not R Astron Soc 93: 325-339.

MERZBACHER E. 1961. Quantum Mechanics. J Wiley \& Sons, Inc., New York, p. 372.

MisNer CM, THORNE KS AND WHEELER JA. 1973. Gravitation. Freeman, San Francisco.

NoERdLinger PD AND PETROSIAN V. 1971. The Effect of the Cosmological Expansion on Self-Gravitating Ensembles of Particles. Astrophys J 168: 1-9.

PAGE DN. 1993. No superluminal expansion of the universe. arXiv:9303008.

PEACOCK JA. 1999. Cosmological Physics. Cambridge Universitary Press, Cambridge.

PEACOCK JA. 2002. An introduction to the physics of cosmology. In Modern Cosmology Bonometto S Gorini V and Moschella U (Eds), IOP, Bristol, p. 9.

PEEBLES PJE. 1980. The Large-Scale Structure of the Universe. Princeton Series in Physics, Princeton University Press, New Jersey, p. 39.

PRICE RH. 2005. In an expanding universe, what doesn't expand? arXiv:0508052.

SCHUCKING E. 1954. Das Schwarzschildsche Linienelement und die Expansion des Weltalls. Zeitschrift fur Physik 137: $595-603$.

SERENO M AND JETZER P. 2007. Evolution of gravitational orbits in the expanding universe. Phys Rev D75, \# 064031, arXiv:0703121.

SLIPHER V. 1915. Spectrographic Observations of Nebulae. Pop Astron 23: 21-24.

WhITING AB. 2004. The expansion of space: Free particle motion and the cosmological redshift. The Observatory 124: 174-189.

ZWICKY F. 1929. On the red shift of spectral lines through the interstellar space. P Natl Acad Sci 15: 773-779. 
